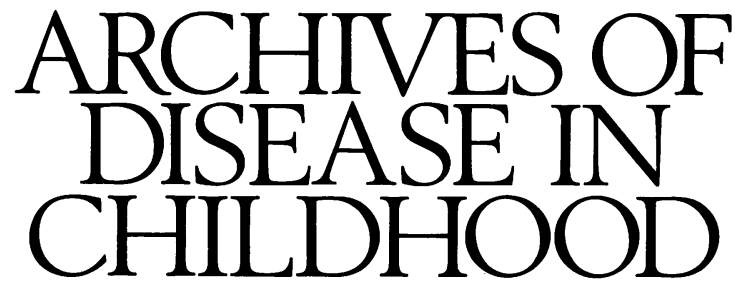

The Fournal of the British Paediatric Association

\title{
Annotations
}

\section{Pathogenesis and management of aspergillosis in cystic fibrosis}

Aspergillus is a common genus of mould that grows on decaying vegetable matter, liberating its spores into the air all the year round but especially in the winter months. The spores are only about 3 microns diameter and can easily penetrate the bronchial tree. Where there is obstructive airways disease and impaired clearance mechanisms, as in cystic fibrosis, the fungus can proliferate and may be cultured from the sputum occasionally. In many patients this does not appear to cause any symptoms but in some it may lead to significant lung disease. The main species causing problems is Aspergillus fumigatus. It may colonise preexisting lung cavities, especially those due to old tuberculosis, producing an aspergilloma. This leads to chronic cough and haemoptyses and radiographically presents as a mass surrounded by a crescent of air in a thick walled cavity. The fungus can spread rapidly in immunocompromised patients leading to an invasive aspergillosis which may prove rapidly fatal. Both these forms of infection have been described in patients with cystic fibrosis but are very unusual. The major problem in cystic fibrosis is allergic bronchopulmonary aspergillosis in which tissue damage is due to the immune reaction of the host.

\section{Allergic bronchopulmonary aspergillosis}

The first patients described were adults with asthma who developed recurrent episodes of pulmonary infiltrates and segmental or lobar collapse, associated with eosinophilia and the production of brown sputum 'plugs' containing A fumigatus. ${ }^{1}$ These episodes appeared to be followed by the development of proximal bronchiectasis at the site of the infiltrates. $^{2}$ There is evidence of both type I and type III immune responses with the production of IgE and IgG antibodies. ${ }^{3}$ The pathology is that of bronchiectasis with inspissated mucus containing fungal hyphae, eosinophils,

Criteria for the diagnosis of allergic bronchopulmonary aspergillosis

Reversible bronchoconstriction
Blood eosinophilia
Pulmonary infiltrate(s)
Positive immediate skin test reaction to A fumigatus extract
Raised serum IgE concentration
Specific IgE to A fumigatus by radioallergosorbent test (RAST
Positive serum precipitins to $A$ fumigatus
Central bronchiectasis
Positive sputum culture for A fumigatus
Brown plugs in sputum
Late skin test reaction

and mononuclear cells in the bronchi-the condition called mucoid impaction. The hyphae do not invade the tissue but may be surrounded by necrosis or chronic inflammation and areas of eosinophilic pneumonia. ${ }^{4}$ Lung biopsy is not needed to make the diagnosis, which can be established by demonstrating six of the criteria shown in the table. ${ }^{5}$

\begin{abstract}
Diagnosis of allergic bronchopulmonary aspergillosis in cystic fibrosis

Allergic bronchopulmonary aspergillosis was first described in two children with cystic fibrosis in $1965 .^{\circ}$ Since then it has become a well recognised complication of cystic fibrosis with an estimated incidence of up to $11 \%{ }^{78}$ Allergic bronchopulmonary aspergillosis is possibly underdiagnosed as the underlying disease itself may be associated with exacerbations of cough and wheeze and there may be infiltrates on the chest radiograph. In practice, it is often diagnosed in patients with cystic fibrosis when an infiltrate fails to clear with antibiotic treatment appropriate to bacteria cultured from the sputum, and it should always be considered in this situation. The associated bronchial obstruction may lead to massive collapse of a lobe or whole lung. ${ }^{7}$ The radiographic findings may be of single or multiple infiltrates, often in the upper lobes. The lesions appear solid but computed tomography may show cavitation. They can occur in patients with any severity of cystic fibrosis including those with mild disease. Some patients have few, if any, symptoms-a recent child seen personally was found to have a large unsuspected opacity when a radiograph was taken for trauma. It is interesting to speculate how often such lesions may occur and resolve undiagnosed and what part they may play in the development of bronchiectasis.
\end{abstract}

\section{Atopy and cystic fibrosis}

Further problems arise in the diagnosis of allergic bronchopulmonary aspergillosis in cystic fibrosis, as in asthma, because of the known tendency of these patients to show some immunological response to $A$ fumigatus. Several cross sectional studies of groups of patients with cystic fibrosis have shown that about one third have circulating precipitins, ${ }^{9}$ and a similar number have positive skin tests to $A$ fumigatus, the incidence tending to be higher in older children and those with more severe disease. ${ }^{111}$ The association of wheezing and of other atopic conditions such as hay fever and eczema is variable, as is a family history of atopy. Many patients with cystic fibrosis do wheeze, 
especially with infections, but often have a poor bronchodilator response and lack the classical bronchoconstriction after exercise that is seen in children with asthma. ${ }^{11}$ Bronchial provocation tests with $A$ fumigatus extract in a small number of patients with cystic fibrosis and with positive skin tests, however, gave a positive immediate result in four patients, two of whom had a dual reaction and had troublesome cough and wheeze for some days after the test. ${ }^{12}$ This suggests that $A$ fumigatus may be causing appreciable symptoms in some patients with cystic fibrosis without producing the complete picture of allergic bronchopulmonary aspergillosis, which may parallel the seropositive phase, without radiographic evidence of lung damage, that is described in asthma. ${ }^{13}$

\section{Management and outcome}

Allergic bronchopulmonary aspergillosis that has been proved should be treated with oral steroids. Prednisolone $0.5-1 \mathrm{mg} / \mathrm{kg} / \mathrm{day}$ leads to rapid resolution of symptoms and of the opacity and a fall in the total and specific IgE concentrations within weeks. ${ }^{5}$ Some authors recommend continuing treatment at a lower dose for a month, six months, or longer until the IgE concentration plateaus, but there are no controlled trials or long term studies comparing different regimes in cystic fibrosis. Inhaled steroids or sodium cromoglycate may improve symptoms but do not prevent recurrent infiltrates, ${ }^{14}$ which are very variable in frequency and severity. There are no long term follow up studies of patients with cystic fibrosis and allergic bronchopulmonary aspergillosis, but in a group of 12 children who had it with asthma there were seven exacerbations detected in three patients over six years. ${ }^{15}$ Personal experience of a few patients and anecdotal reports from others suggest that some patients with cystic fibrosis have recurrences of allergic bronchopulmonary aspergillosis over two or three years but tend to settle after that time and do well in the long term-or, at least, no worse than other patients with cystic fibrosis. Bronchiectasis, often with the 'fingerglove' appearance of mucoid impaction, is usually apparent radiographically when the opacity clears but in time becomes much less prominent and tends to merge into the background lung disease so that it is difficult to know how much it has contributed. Severe pulmonary damage has been described in adults with asthma, ${ }^{16}$ however, and there is clearly a risk that this may occur in cystic fibrosis, leading to an increased rate of loss of lung function.

There is sometimes reluctance to use steroids in cystic fibrosis because of the presence of bacterial infection, but in practice it does not seem to lead to spread of infection and there is some evidence that it may actually be helpful by suppressing damaging immune reactions. ${ }^{17}$ Long term steroid treatment, however, has well known complications and posterior subcapsular cataracts have recently been described in patients with cystic fibrosis on prednisone for allergic bronchopulmonary aspergillosis, which appeared unrelated to the dose or duration of treatment. ${ }^{18}$

Antifungal drugs have not been used in the past because inhaled treatment, such as dyes and natamycin, seemed ineffective and allergic bronchopulmonary aspergillosis was not thought to warrant the side effects of amphotericin. With the introduction of oral drugs such as ketoconazole and itraconazole, interest in this form of treatment revived and there have been occasional reports of benefit in indi- vidual patients. A small controlled trial of ketoconazole in patients without cystic fibrosis but with allergic bronchopulmonary aspergillosis showed an improvement in symptoms and fall in specific IgG during treatment compared with placebo. ${ }^{19}$ No side effects were seen, but the trial was abandoned when reports of serious liver damage due to ketoconazole were published. ${ }^{20}$ Very little information is available yet about itraconazole. For the present the use of antifungal drugs does not seem to be warranted as a rule but might be considered in a patient who has significant steroid side effects or uncontrolled symptoms.

Avoidance of $A$ fumigatus does not seem feasible though it is wise to make sure that nebulisers and other equipment are kept clean and dry to prevent fungal contamination. Of five children with allergic bronchopulmonary aspergillosis recently reported from Dublin, however, only one was using a home nebuliser. ${ }^{8}$ Its incidence does not seem to increase significantly in older patients with cystic fibrosis. It is a condition that needs to be considered regularly, investigated appropriately, and treated promptly with steroids, both to improve symptoms, if any, and to prevent long term lung damage.

\section{E J HILLER}

City Hospital,

Hucknall Road

Nottingham NGS IPB

I would like to thank Dr Denis Shale for helpful advice.

Hinson KFW, Moon AJ, Plummer NS. Bronchopulmonary aspergillosis. Thorax 1952;7:317-33.

2 McCarthy DS, Simon G, Hargreave FE. The radiological appearances in bronchopulmonary aspergillosis. Clin Radiol 1970;21:366-75.

3 Wang JLF, Patterson R, Rosenberg M, et al. Serum IgE and IgG antibody activity against Aspergillus fumigatus as a diagnostic aid in allergic bronchoactivity against Aspergillus fumigatus as a diagnostic aid in allergic

4 Suavin RG, Bedrossian CW, Hutcheson PS, et al. A pathologic study of allergic bronchopulmonary aspergillosis. F Allergy Clin Immunol 1988;81: gic bro $718-25$.

5 Rosenberg M, Patterson R, Mintzer R, et al. Clinical and immunologic criteria for the diagnosis of allergic bronchopulmonary aspergillosis. Ann Intern Med 1977;86:405-14.

6 Mearns M, Young W, Batten J. Transient pulmonary infiltrations in cystic fibrosis due to allergic aspergillosis. Thorax 1965;20:385-92.

7 Brueton MJ, Ormerod LP, Shah KJ, Anderson CM. Allergic bronchopulmonary aspergillosis complicating cystic fibrosis in childhood. Arch $D i$ Child 1980;55:348-53.

8 Maguire S, Moriarty P, Tempany E, Fitzgerald M. Unusual clustering of allergic bronchopulmonary aspergillosis in children with cystic fibrosis. Pediatrics 1988;82:835-9.

9 Mearns M, Longbottom J, Batten J. Precipitating antibodies to A fumigatu in cystic fibrosis. Lancet 1967;i:538-9.

10 Warner JO, Taylor BW, Norman AP, Soothill JF Association of cystic fibrosis with allergy. Arch Dis Child 1976;51:507-11.

11 Silverman $M$, Hobbs FDR, Gordon IRS, Carswell F. Cystic fibrosis, atopy and airways lability. Arch Dis Child 1978;53:873-7.

12 Price JF, Weller PH, Harper SA, Matthews DJ. Response to bronchial provocation and exercise in children with cystic fibrosis. Clin Allergy 1979; provocatio

13 Patterson R, Greenberger PA, Halwig JM, et al. Allergic bronchopulmonary aspergillosis - natural history and classification of early disease by serologic and roentgenographic studies. Arch Intern Med 1986;146:916-8.

14 Crompton GK. Inhaled beclomethasone dipropronate in allergic bronchopulmonary aspergillosis. Br $\mathcal{J}$ Dis Chest 1979;73:349-56.

15 Wang JL, Patterson R, Mintzer R, et al. Allergic bronchopulmonary aspergillosis in pediatric practise. $\mathcal{F}$ Pediatr 1979;94:376-81

16 Lee TM, Greenberger PA, Patterson R, et al. Stage V (fibrotic) allergic bronchopulmonary aspergillosis. Arch Intern Med 1987;147:319-23.

17 Auerbach HS, Williams M, Kirkpatrick JA, Colten HR. Alternate day prednisone reduces morbidity and improves pulmonary function in cystic fibrosis. Lancet 1985;ii:686-8.

18 Majure M, Mroueh S, Spock A. Risk factors for the development of posterio subcapsular cataracts in patients with cystic fibrosis and allergic bronchopulmonary aspergillosis treated with corticosteroids. Pediatr Pulmonol 1989; 6:260-2.

19 Shale DJ, Faux JA, Lane DJ. Trial of ketoconazole in non-invasive pulmonary aspergillosis. Thorax 1987;42:26-31.

20 Lewis JH, Zimmerman HJ, Benson GD, Ishak KG. Hepatic injury associated with ketoconazole therapy - analysis of 33 cases. Gastroenterology 1984;86: w03-13. 